Original paper

\title{
The optimal balance between quality and efficiency in proton radiography imaging technique at various proton beam energies: A Monte Carlo study
}

\author{
A.K. Biegun ${ }^{\text {a,* }}$, M-J. van Goethem ${ }^{\mathrm{a}, \mathrm{b}}$, E.R. van der Graaf ${ }^{\mathrm{a}}$, M. van Beuzekom ${ }^{\mathrm{c}}$, E.N. Koffeman ${ }^{\mathrm{c}}$, \\ T. Nakaji ${ }^{\mathrm{d}}$, J. Takatsu ${ }^{\mathrm{d}}$, J. Visser $^{\mathrm{c}}$, S. Brandenburg ${ }^{\mathrm{a}}$ \\ ${ }^{a}$ KVI-Center for Advanced Radiation Technology, University of Groningen, Zernikelaan 25, 9747AA Groningen, The Netherlands \\ ${ }^{\mathrm{b}}$ Department of Radiation Oncology, University Medical Center Groningen, University of Groningen, Hanzeplein 1, 9700 RB Groningen, The Netherlands \\ ${ }^{\mathrm{c}}$ National Institute for Subatomic Physics (Nikhef), Science Park 105, 1098 XG Amsterdam, The Netherlands \\ ${ }^{\mathrm{d}}$ Department of Radiation Oncology, Graduate School of Medicine, Osaka University, 2-2 D10 Yamadaoka, Suita, Osaka 565-0871, Japan
}

\section{A R T I C L E I N F O}

\section{Article history:}

Received 16 January 2017

Received in Revised form 4 August 2017

Accepted 9 August 2017

Available online 30 August 2017

\section{Keywords:}

Proton radiography

Proton scattering angle

Proton treatment plan

\begin{abstract}
A B S T R A C T
Proton radiography is a novel imaging modality that allows direct measurement of the proton energy loss in various tissues. Currently, due to the conversion of so-called Hounsfield units from X-ray Computed Tomography (CT) into relative proton stopping powers (RPSP), the uncertainties of RPSP are 3-5\% or higher, which need to be minimized down to $1 \%$ to make the proton treatment plans more accurate.

In this work, we simulated a proton radiography system, with position-sensitive detectors (PSDs) and a residual energy detector (RED). The simulations were built using Geant4, a Monte Carlo simulation toolkit. A phantom, consisting of several materials was placed between the PSDs of various Water Equivalent Thicknesses (WET), corresponding to an ideal detector, a gaseous detector, silicon and plastic scintillator detectors. The energy loss radiograph and the scattering angle distributions of the protons were studied for proton beam energies of $150 \mathrm{MeV}, 190 \mathrm{MeV}$ and $230 \mathrm{MeV}$. To improve the image quality deteriorated by the multiple Coulomb scattering (MCS), protons with small angles were selected. Two ways of calculating a scattering angle were considered using the proton's direction and position.

A scattering angle cut of $8.7 \mathrm{mrad}$ was applied giving an optimal balance between quality and efficiency of the radiographic image. For the three proton beam energies, the number of protons used in image reconstruction with the direction method was half the number of protons kept using the position method.

(C) 2017 Associazione Italiana di Fisica Medica. Published by Elsevier Ltd. This is an open access article under the CC BY-NC-ND license (http://creativecommons.org/licenses/by-nc-nd/4.0/).
\end{abstract}

\section{Introduction}

The quality of cancer treatment with protons critically depends on the accurate determination of proton stopping powers (PSP) of the tissues traversed by the protons. Proton treatment planning is based on stopping powers derived from CT images leading to systematic uncertainties of $3-5 \%$ and even up to $10 \%$ in regions containing bone [1-4]. This may result in a lower dose being deposited in parts of the tumor and/or an overdose in healthy tissues.

The use of proton radiography, which gives direct access to PSPs, can reduce the uncertainty in the translation of the X-ray CT image into a map of PSPs.

\footnotetext{
* Corresponding author.

E-mail address: a.k.biegun@rug.nl (A.K. Biegun).
}

Several strategies for proton radiography and its 3D extension proton Computed Tomography ( $\mathrm{pCT}$ ) are being explored by various laboratories [5-10]. Measuring the energy loss and scattering angle of an individual proton passing through the patient is the best way to obtain a sufficiently accurate radiograph of the phantom (patient). For these measurements, the initial proton beam energy should be higher than that chosen for patient treatment to ensure that the protons leave the patient's body. From the entry and exit points and angles of each proton, combined with the deposited energy, the most likely path can be calculated for each proton. This, together with already available CT data, can improve the accuracy of the PSP prediction, and thereby improve the quality of the proton treatment plan.

At KVI-CART we performed proton radiography (pRG) experiments using a novel prototype of a gaseous PSD (Timepix-based Time Projection Chamber, TPC), developed by Nikhef $[8,11]$. In these experiments, we used a proton beam energy of $150 \mathrm{MeV}$ to 
irradiate a phantom. The experiments were simulated with Geant4. The comparison of both experimental and simulated data was in excellent agreement, which was a motivation for further studies [8].

The challenge in PRG imaging arises from the MCS of the protons traversing different materials causing blurring of the radiography image. Thus, to improve the image quality and identify each material in the phantom, cuts for the proton scattering angle have to be tuned carefully.

In this paper we present the analysis of two different proton scattering angle calculations, based on direction and position of a proton, and its effect on energy loss radiographs and statistics. The analysis was performed for detectors with different WET, these include: an ideal massless detector, a gaseous detector, silicon ( $\mathrm{Si}$ ) and plastic scintillator detectors. The proton energies chosen for the simulations were $150 \mathrm{MeV}, 190 \mathrm{MeV}$ and $230 \mathrm{MeV}$.

\section{Material and methods}

\subsection{Monte Carlo simulations}

The Monte Carlo (MC) simulations discussed in this paper were performed with Geant4 version 9.6.p03 [12]. A scheme of the simulation setup implemented in our code is sketched in Fig. 1.

Each of $5.0 \cdot 10^{7}$ generated protons passed through the two PSDs before and after the phantom, and it was stopped in the RED. The proton beam field was generated at a distance of $100 \mathrm{~cm}$ from the PSD1 with the size of $6 \mathrm{~cm} \times 10 \mathrm{~cm}$, in $\mathrm{x}$ and $\mathrm{y}$ direction, respectively (Fig. 1). No divergence of the beam was applied. Simulations were performed for the WET of each of the PSDs, thus mimicking the various detector types that have been used in $\mathrm{pRG/pCT}$ systems with tracking detectors. The list of studied PSD detectors with their specifications is shown in Table 1. Different PSDs types, their materials and thicknesses were taken from setups that were used in proton radiography systems described in the literature [10]. The thickness of the gaseous detector was taken based on the system that we previously used in our experiments at KVI-CART [8], the thickness of the Si detector was taken from the PRIMA I experiment [7], and the thickness of the plastic scintillator was taken from the proton radiography setup used at the Paul Scherrer Institute [13]. The RED in all simulations was filled with BaF2, similarly to what was used during our experiments at KVICART.

The proton beam energies used in the simulations are $\mathrm{E}_{\text {beam }}=150 \mathrm{MeV}, 190 \mathrm{MeV}$ and $230 \mathrm{MeV}$. The energy loss of a proton in the phantom, $\Delta E$, was calculated as a difference between the known proton beam energy, $E_{\text {beam }}$, and the residual energy, $E_{R E D}$, detected in RED:

$\Delta E=E_{\text {beam }}-E_{R E D}$

The reference physics list used in our simulations was QGSP_BIC_EMY, which has been suggested by Cirrone et al. [14] to obtain a high level of accuracy. This physics list has been optimized for hadron radiotherapy simulations.

\subsection{Phantom}

The phantom is composed of a block of polymethyl methacrylate (PMMA), in which six cylinders of various sizes and materials, including tissue surrogates, were inserted (Fig. 2, left).

Three of the cylinders contain only one tissue surrogate material, such as: liver (In1), breast (In3) and lung (In4) and the other three inserts simulate an air cavity (In2), a lung tumour (In5) and dental fillings (In6). The diameters of the implemented cylinders are $8,10,12,15$, and $20 \mathrm{~mm}$ for In3, In2, In6, In 1 , and In 4 and In5, respectively. Additionally, the topmost cylinder (In6) is inserted at an angle of 60 degrees with respect to the z-axis (beam direction).

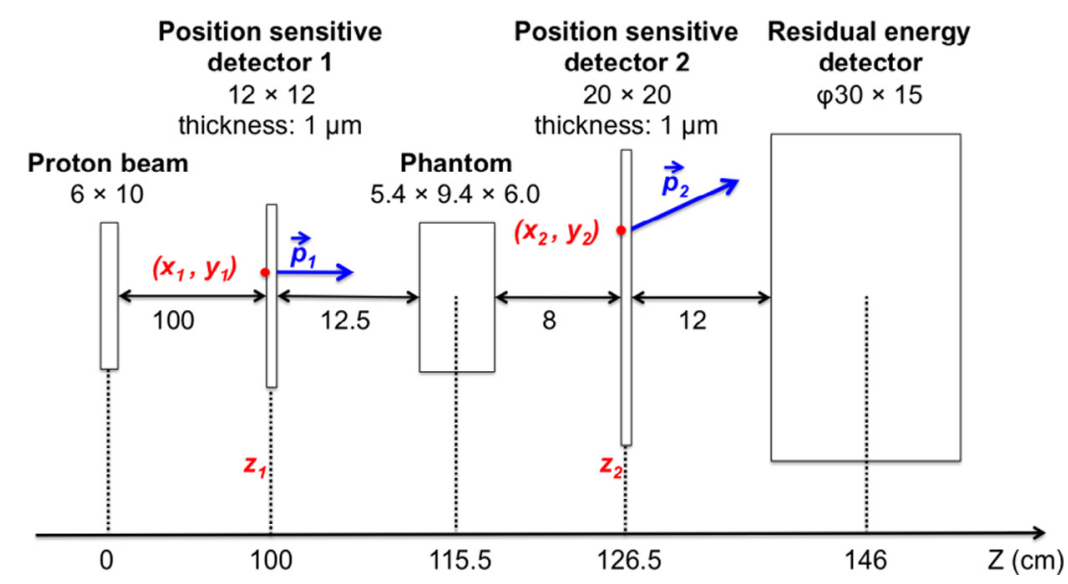

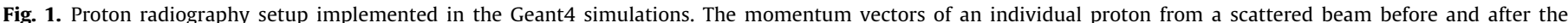

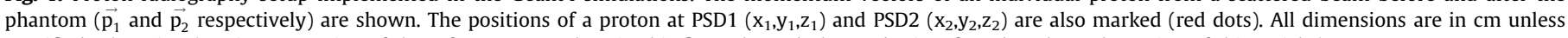
specified otherwise. (For interpretation of the references to colour in this figure legend, the reader is referred to the web version of this article.)

Table 1

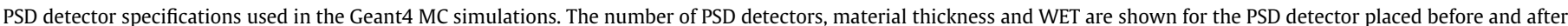
the phantom.

\begin{tabular}{|c|c|c|c|c|c|}
\hline PSD detector type & Number of PSD detectors & Material & Physical density $\left(\mathrm{g} / \mathrm{cm}^{3}\right)$ & Material thickness (mm) & WET $(\mathrm{mm})$ \\
\hline Ideal & 1 & Air & 0.0012 & 0.001 & - \\
\hline Gaseous (TPC)[8] & 1 & Isobutene $\mathrm{C}_{4} \mathrm{H}_{10}$ & 0.0025 & 30.0 & 0.394 \\
\hline Silicon[7] & 2 & Silicon & 2.330 & 0.4 & 0.752 \\
\hline Plastic scintillator[13] & 2 & Bicron BCF12 & 1.032 & 4.0 & 4.106 \\
\hline
\end{tabular}




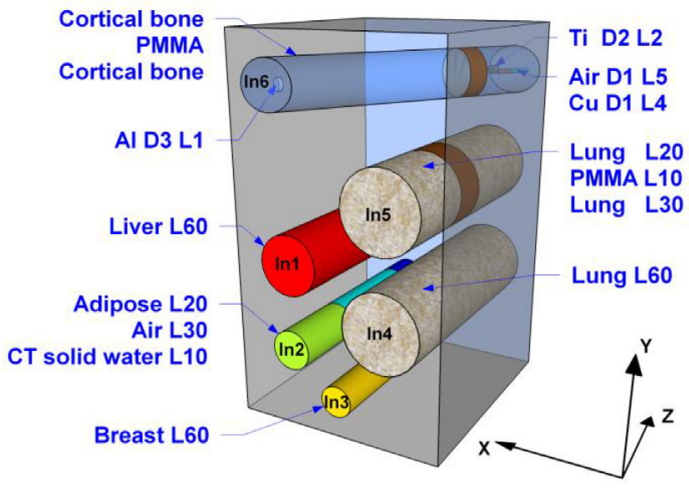

\begin{tabular}{|c|c|}
\hline Phantom material & $\begin{array}{c}\text { Physical density } \\
\left(\mathrm{g} / \mathrm{cm}^{3}\right)\end{array}$ \\
\hline Lung $^{*}$ & 0.428 \\
\hline Adipose (fat) $^{*}$ & 0.946 \\
\hline Brest $^{*}$ & 0.981 \\
\hline Liver $^{*}$ & 1.095 \\
\hline CT solid water $^{*}$ & 1.045 \\
\hline Cortical bone* $^{*}$ & 1.823 \\
\hline Air & 0.0012 \\
\hline PMMA & 1.180 \\
\hline $\mathrm{Al}$ & 2.699 \\
\hline $\mathrm{Ti}$ & 4.510 \\
\hline $\mathrm{Cu}$ & 8.960 \\
\hline
\end{tabular}

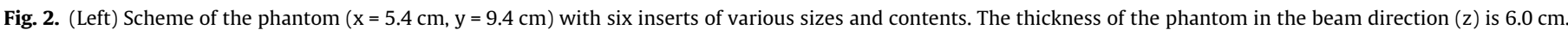

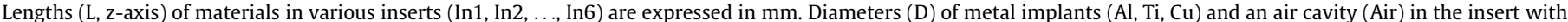

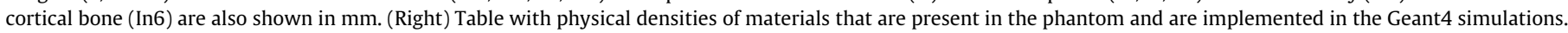
Materials marked as * represent Gammex tissue surrogates [15].

\subsection{Calculations of proton scattering angles}

In proton radiography the images are blurred because of MCS. Thus, it is important to find the best way to reduce the impact of MCS on the extracted proton energy loss radiographic image to minimize the blurring and, consequently, to improve the accuracy of the energy loss map. The scattering angle of a proton was calculated using both the direction and the positions of a proton before and after traversing the phantom, according to the following formulas:

$\phi_{12}^{\vec{p}}(\mathrm{rad})=\cos ^{-1} \frac{\vec{p}_{1} \vec{p}_{2}}{\left|\vec{p}_{1}\right|\left|\vec{p}_{2}\right|}$

where: $\vec{p}_{1}\left(\mathrm{p}_{\mathrm{x} 1}, \mathrm{p}_{\mathrm{y} 1}, \mathrm{p}_{\mathrm{z} 1}\right)$ and $\vec{p}_{2}\left(\mathrm{p}_{\mathrm{x} 2}, \mathrm{p}_{\mathrm{y} 2}, \mathrm{p}_{\mathrm{z} 2}\right)$ are the proton directions defined with momentum vectors at PSD1 and PSD2, respectively (Fig. 1).

$\phi_{12}^{p o s}(\mathrm{rad})=\tan ^{-1} \frac{\sqrt{\left(x_{2}-x_{1}\right)^{2}+\left(y_{2}-y_{1}\right)^{2}}}{\left(z_{2}-z_{1}\right)}$

where: $x_{1}, y_{1}, z_{1}$ and $x_{2}, y_{2}, z_{2}$ represent the proton positions at PSD1 and PSD2, respectively. This represents a "zero order" approximation.

\section{Results and discussion}

Fig. 3 shows the proton scattering angle distributions determined from the proton direction, $\theta_{12}^{\vec{p}}$ (Fig. 3a) and the proton position, $\theta_{12}^{\text {pos }}$ (Fig. 3b), respectively, according to (2) and (3). The angular distributions are shown for $E_{\text {beam }}=150 \mathrm{MeV}$. The PSD1 and PSD2 were filled with water and the corresponding WET is shown in Table 1. The scattering angle determined from the proton direction (Fig. 3a) shows that a significant number of protons travel in the forward direction, with a minimum scattering, while the distributions based on the proton position (Fig. 3b) is broader.

Various cuts on the scattering angle were applied in order to have a sharper image and to better distinguish the various insertions in the object, as discussed in our previous study [9]. The best trade-off between sharp transitions of various materials in the phantom and the statistics was found for scattering angles smaller than 8.7 mrad. Therefore, this scattering angle was also used in this analysis.

Fig. 4 shows the percentage of protons surviving the $8.7 \mathrm{mrad}$ cut, when proton direction (Fig. 4a) and position (Fig. 4b) are used to make the cut. Using the proton position to calculate scattering angles $\theta_{12}^{\text {pos }}$, more protons, by a factor of two, are accepted to build the radiographic image. Based on the results from [9] we verified
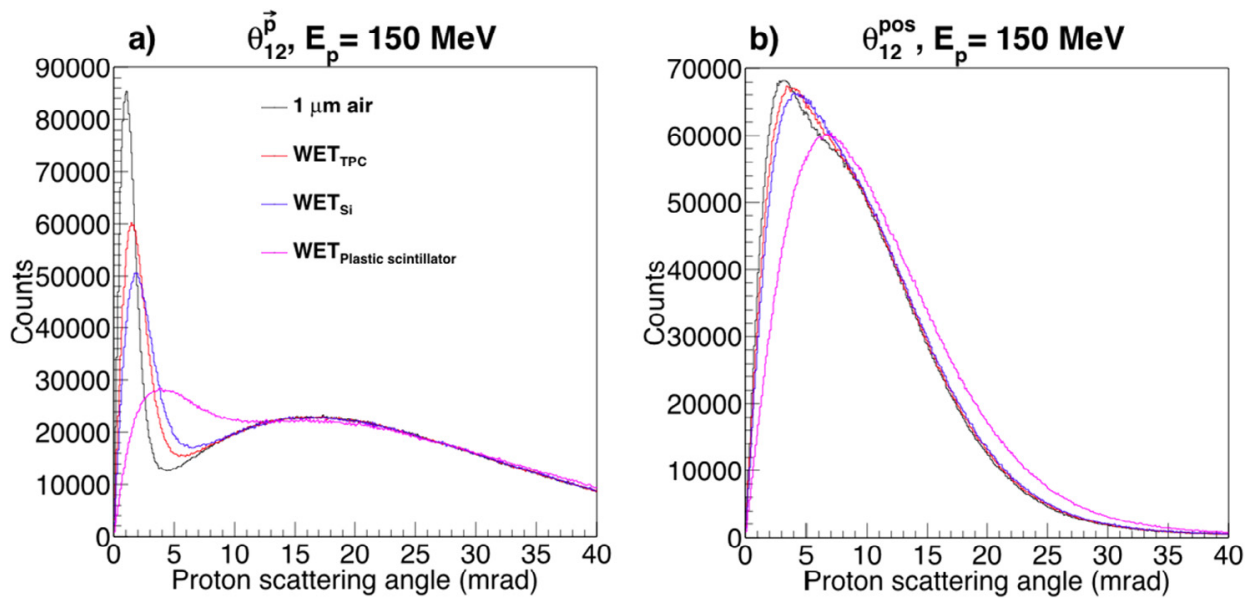

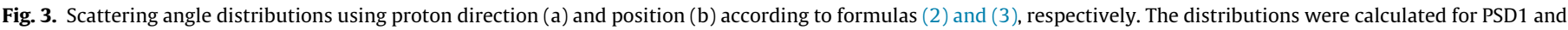
PSD2 with various WET (Table 1) with proton beam energy of $150 \mathrm{MeV}$. 

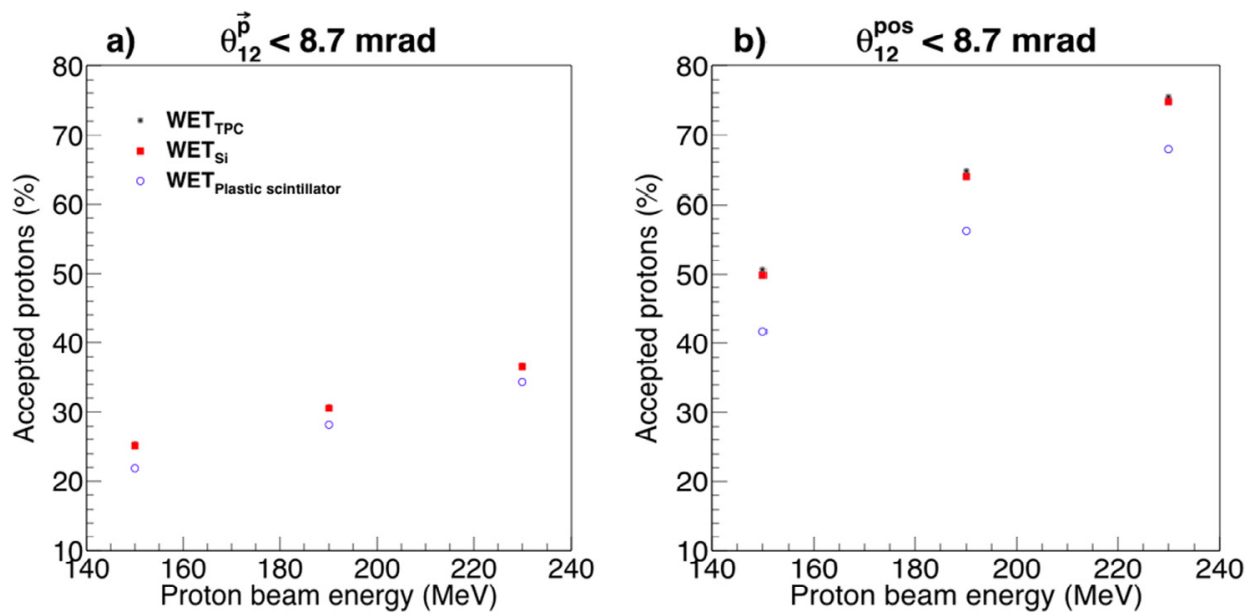

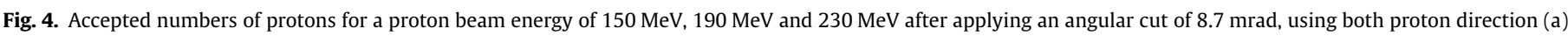
and position (b).

a) $1 \mu \mathrm{m}$ Air, $\mathrm{E}_{\mathrm{p}}=150 \mathrm{MeV}_{(\mathrm{MeV})}^{<\Delta E>}$

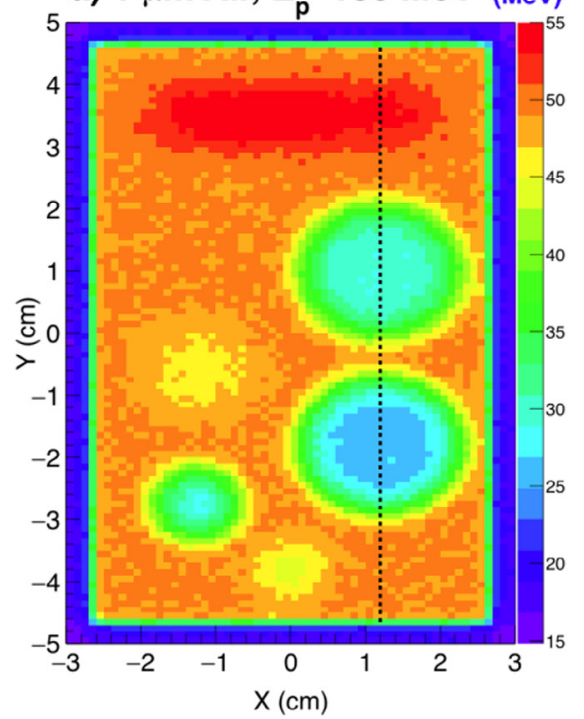

c) $\mathrm{WET}_{\mathrm{si}}, \mathrm{E}_{\mathrm{p}}=\mathbf{1 5 0 \mathrm { MeV }}$

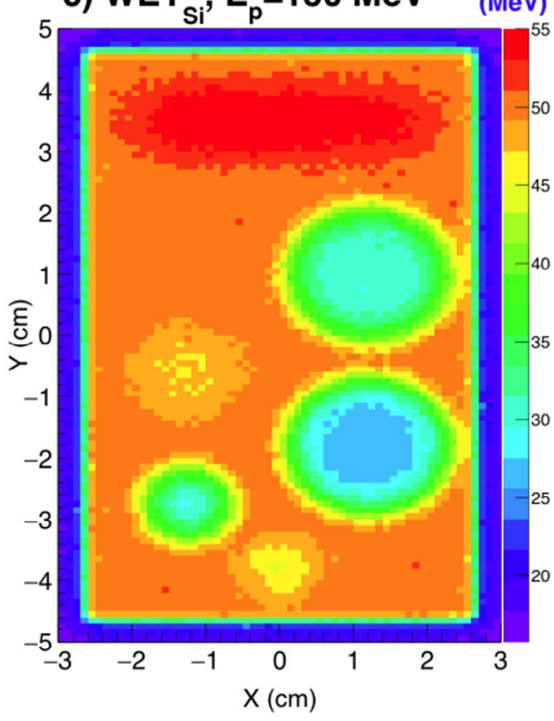

b) $\mathrm{WET}_{\mathrm{TPC}}, \mathrm{E}_{\mathrm{p}}=150 \mathrm{MeV} \stackrel{<\mathrm{MEV}>}{(\mathrm{MeV})}$

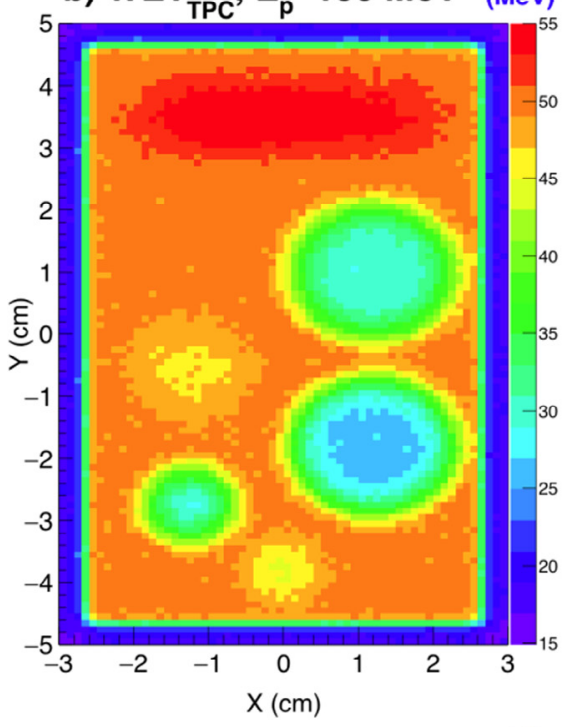

d) WET $T_{\text {PI.Sci }}, E_{p}=150 \mathrm{MeV}_{(\mathrm{MeV})}^{<\Delta E>>}$

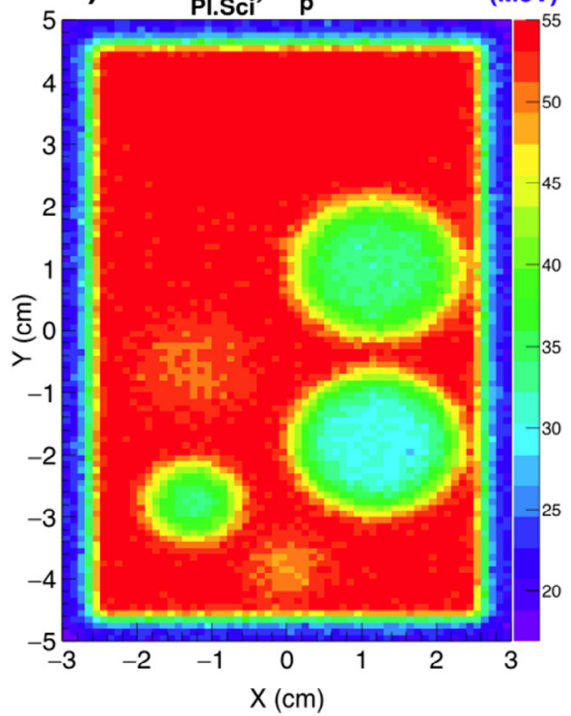

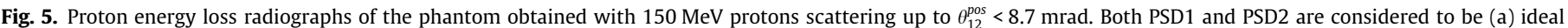

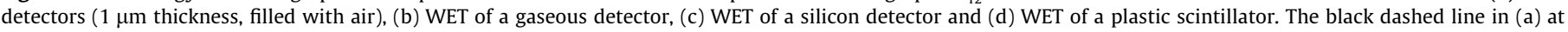
$\mathrm{X}=1.15 \mathrm{~cm}$ shows the position of the projection in Fig. 6 . 
that the results for $\mathrm{E}_{\text {beam }}=150 \mathrm{MeV}$ are reproducible. In addition, the number of protons surviving the cut for higher proton beam energies of $190 \mathrm{MeV}$ and $230 \mathrm{MeV}$ were also determined and shown in Fig. 4.

Using the proton position, the number of accepted protons is nearly $50 \%$ and increases to $65 \%$ and $75 \%$ for $E_{\text {beam }}=150 \mathrm{MeV}$, $190 \mathrm{MeV}$ and $230 \mathrm{MeV}$, respectively (Fig. 4b), while using proton direction (Fig. 4a), these numbers correspond to $25 \%, 31 \%$ and $37 \%$ for $E_{\text {beam }}=150 \mathrm{MeV}, 190 \mathrm{MeV}$ and $230 \mathrm{MeV}$. Thus, the statistics shows that it is better to use the proton position method to calculate the proton scattering angle.

Fig. 5 shows radiographic images of the phantom reconstructed based on $\theta_{12}^{\text {pos }}$ of a proton that passed through all the detectors and that scattered up to $8.7 \mathrm{mrad}$. The proton energy loss, $\Delta E$, is calculated using (1).

Fig. 5a represents the energy loss radiograph when both PSDs were filled with air (ideal detectors), while Fig. 5b, c and d represent PSDs filled with WET of TPC, Si and plastic scintillators (Table 1). Fig. 5a also indicates with the position of the dashed line, located at $X=1.15 \mathrm{~cm}$, for which the projections in Fig. 6a are plotted. The reconstructed energy loss radiographs for PSDs filled with air and WET of TPC are very comparable, Fig. 5a and b, with all inserts visible. When PSDs are filled with Si (Fig. 5c), a part of the radiographic image is blurred for inserts with cortical bone, liver and breast (see Fig. 2 for the reference), while these three inserts are hardly recognizable when PSDs are filled with plastic scintillator; the radiographic image in Fig. $5 d$ is blurred the most.

Fig. 6a demonstrates the differences in the proton energy loss profiles through selected material of the phantom for various WET of PSDs (Table 1) and the transitions between materials.

$\Delta E$ profiles of the radiographic image were calculated in correspondence of the dashed line in Fig. $5 \mathrm{a}$ and were obtained with $150 \mathrm{MeV}$ protons scattering up to $8.7 \mathrm{mrad}\left(\theta_{12}^{\text {pos }}\right)$. It can be observed that the transition between various phantom materials is least steep for the WET of plastic scintillators. This is expected, because the WET of plastic scintillators is the largest (Table 1). For both the WET of TPC and Si, sharp transitions between materials are visible (Fig. 6a). The thickness of gas is significantly larger than the one of the Si and only $0.4 \mathrm{~mm}$ of Si has been considered in the simulations for each PSD. Furthermore, no visible difference in sharpness between PSDs filled with air (ideal detector) and the WET of TPC is observed, which is expected because the WET of TPC is very small $(<0.4 \mathrm{~mm})$. For higher proton beam energies, $190 \mathrm{MeV}$ and $230 \mathrm{MeV}$, the differences in $\Delta E$ profiles between various PSD materials are smaller, as expected due to decreasing MCS of protons with increasing proton beam energy.

Fig. $6 \mathrm{~b}$ presents the energy loss ratio ( $\Delta E$ ratio) between the energy loss measured with TPC, Si, plastic scintillator and air, for $150 \mathrm{MeV}$ protons. The $\Delta E$ ratio for TPC and $\mathrm{Si}$ is comparable and the fluctuation is between 0.95 and 1.1 (15\%), with the average value of 1.02 (determined by visual inspection), whereas the fluctuation for plastic scintillator is larger, between 0.97 and 1.31 (34\%), with an average value of 1.12 .

Similar $\Delta E$ profiles and $\Delta E$ ratios were obtained for the same cut of scattered protons of $8.7 \mathrm{mrad}$, where the scattering angle $\theta_{12}^{\vec{p}}$ was calculated using the proton direction (formula 2). The fluctuation of the profiles and ratios were observed to be larger than in Fig. $6 a$ and $b$, with the average value of 1.03 for protons with energy of $150 \mathrm{MeV}$, and can be seen in Table 2. Furthermore, both $\Delta E$ profiles and $\Delta E$ ratios were obtained for higher proton beam energies of $190 \mathrm{MeV}$ and $230 \mathrm{MeV}$ (not shown) and similar trend was observed. The average values of $\Delta E$ ratios are smaller when scattering angle is calculated using the proton position than the proton direction ( 1.01 for $\theta_{12}^{\text {pos }}$ vs. 1.03 for $\theta_{12}^{\vec{p}}$, for proton beam energies of $190 \mathrm{MeV}$ and $230 \mathrm{MeV}$, respectively). All the average values of $\Delta E$ ratio fluctuations are shown in Table 2.
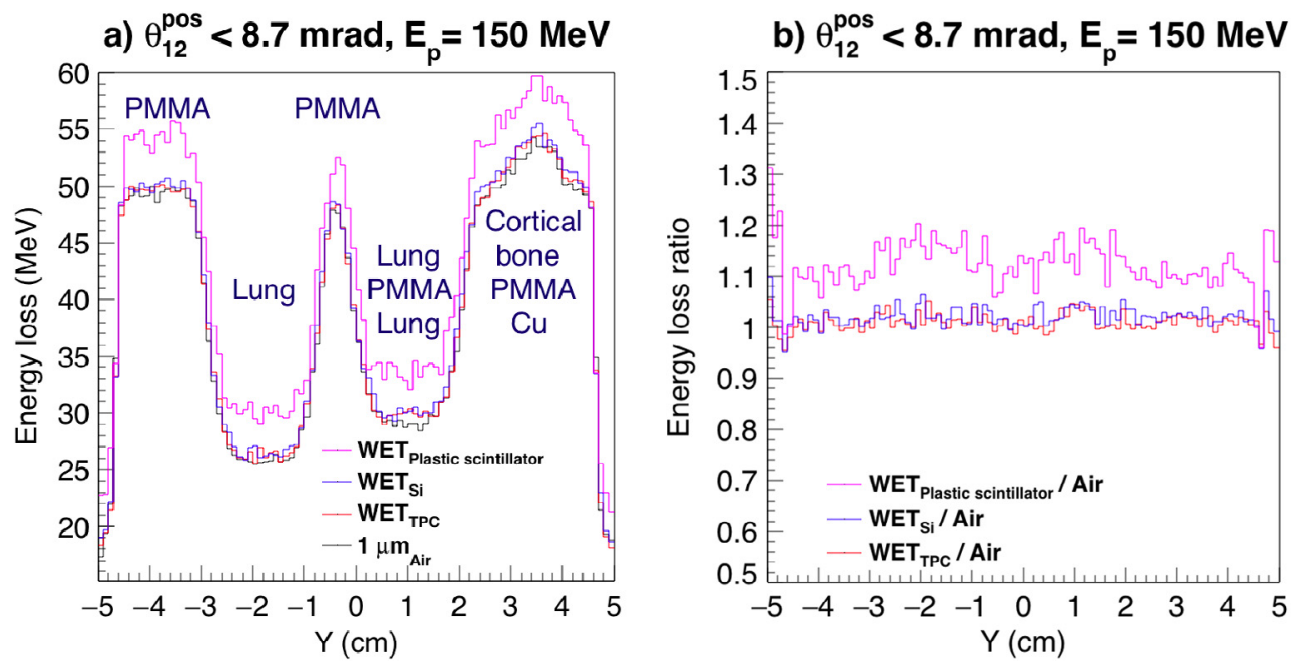

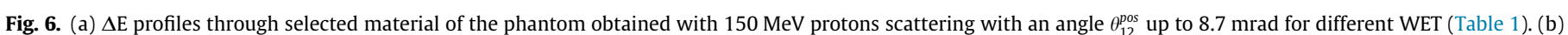
$\Delta \mathrm{E}$ ratio for $150 \mathrm{MeV}$ protons and $\theta_{12}^{\text {pos }}$ up to $8.7 \mathrm{mrad}$ for different WET.

Table 2

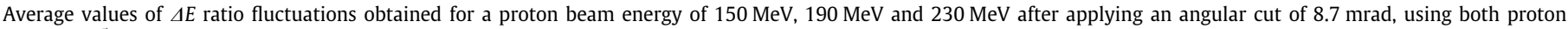
direction $\left(\theta_{12}^{\vec{p}}\right)$ and position $\left(\theta_{12}^{\text {pos }}\right)$.

\begin{tabular}{|c|c|c|c|c|c|c|}
\hline \multirow[t]{2}{*}{$\Delta \mathrm{E}$ ratio (average) } & \multicolumn{3}{|l|}{$\theta_{12}^{\vec{p}}$} & \multicolumn{3}{|l|}{$\theta_{12}^{\text {pos }}$} \\
\hline & $150 \mathrm{MeV}$ & $190 \mathrm{MeV}$ & $230 \mathrm{MeV}$ & $150 \mathrm{MeV}$ & $190 \mathrm{MeV}$ & $230 \mathrm{MeV}$ \\
\hline Gaseous (TPC) & 1.03 & 1.03 & 1.03 & 1.02 & 1.01 & 1.01 \\
\hline Silicon & 1.03 & 1.03 & 1.03 & 1.02 & 1.01 & 1.01 \\
\hline Plastic scintillator scintillator & 1.12 & 1.07 & 1.04 & 1.12 & 1.06 & 1.04 \\
\hline
\end{tabular}




\section{Conclusions}

In this paper we present the results of a study on proton radiography, an imaging technique aiming at a direct (and therefore more accurate) calculation of the PSP of an object under study (patient).

The MCS of protons passing through various materials blurs the energy loss radiographic image. Selecting protons with small scattering angles sharpens the edges between materials in the object, decreasing the blurring. Two ways of calculating the proton scattering angle have been studied, where either the proton direction $\left(\theta_{12}^{\vec{p}}\right)$ or the proton position $\left(\theta_{12}^{\text {pos }}\right)$ was used to calculate the scattering angular distribution. When applying cuts in the radiographic image reconstruction based on $\theta_{12}^{\text {pos }}$, the number of "accepted" protons was doubled compared to when $\theta_{12}^{\vec{p}}$ was used for all three energies, specifically: $51 \%$ vs. $25 \%$ with $\mathrm{E}_{\text {beam }}=150 \mathrm{MeV}, 65 \%$ vs. $30 \%$ with $E_{\text {beam }}=190 \mathrm{MeV}$, and $75 \%$ vs. $37 \%$ with $E_{\text {beam }}=230 \mathrm{MeV}$.

Plastic scintillators, such as PSD1 and PSD2, seem to be the most disadvantageous in the pRG/pCT imaging system, unless the highest proton beam energy of $230 \mathrm{MeV}$ is used. For a more realistic representation of $\mathrm{Si}$ detectors, additional materials should be added to the simulations consisting of, for example, a PCB material to which the Si detector is connected. Protons from the beam pass through the PCB material undergoing MCS and causing further blurring of the radiographic image. Thus, it will make the results for $\mathrm{PSD}_{\mathrm{Si}}$ less accurate than for PSD $\mathrm{TPC}_{\mathrm{TP}}$. Furthermore, the thickness of a single Si detector is usually taken to be at least $300 \mu \mathrm{m}$. As PSDs of at least two (or three in some pRG/pCT prototypes) $\mathrm{Si}$ detectors are placed before and after the object under study, this will increase the image blurring further. The MCS is element dependent and grows with the atomic number of the material. The most promising PSDs in a pRG/pCT system are expected to be gaseous detectors. The MCS in low-density gas is the smallest amongst the discussed materials giving the least expected blurring of the radiographic image. The readout chip in gaseous PSDs can be located at the surface perpendicular to the beam direction, such that protons will not pass through the chip material, but mostly pass through the gas [8]. More simulations and experiments are required to study the MCS on complex phantoms and real tissues.

\section{Acknowledgments}

We would like to thank the Center for Information Technology of the University of Groningen for their support and for providing access to the Peregrine high-performance computing cluster, where the Monte Carlo simulations were performed. This work was partially supported by the Japan Society for Promotion of Science Core-to-Core Program (number 23003).

\section{References}

[1] Schneider U, Pedroni E, Lomax A. The calibration of CT Hounsfield units for radiotherapy treatment planning. Phys Med Biol 1996;41:111-24.

[2] Paganetti H. Range uncertainties in proton therapy and the role of Monte Carlo simulations. Phys Med Biol 2012:57:R99-R117.

[3] Plautz T et al. $200 \mathrm{MeV}$ proton radiography studies with a hand phantom using a prototype proton CT scanner. IEEE Trans Med Imag 2014;33(4):875-81.

[4] Schuemann J et al. Site-specific range uncertainties caused by dose calculation algorithms for proton therapy. Phys Med Biol 2014;59:4007-31.

[5] Schneider U, Pedroni Eros. Multiple Coulomb scattering and spatial resolution in proton radiography. Med Phys 1994;21:1657-63.

[6] Ryu $\mathrm{H}$ et al. Density and spatial resolutions of proton radiography using a range modulation technique. Phys Med Biol 2008;53:5461-8.

[7] Sipala V et al. A proton Computed Tomography system for medical applications. JINST 2013;8:C02021.

[8] Biegun AK et al. Proton radiography with timepix based time projection chambers. IEEE Trans Med Imag 2016;35(4):1099-105.

[9] Biegun AK et al. Proton energy and scattering angle radiographs to improve proton treatment planning: a Monte Carlo study. JINST 2016;11:C12015.

[10] Poludniowski G, Allinson NM, Evans PM. Proton radiography and tomography with application to proton therapy. Br J Radiol 2015;88:20150134 [and references therein].

[11] https://www.nikhef.nl.

[12] Agostinelli S et al. Geant4-a simulation toolkit. Nucl Instr Meth in Phys Res 2003;506:250-303.

[13] Schneider U et al. First proton radiography of an animal patient. Med Phys 2004;31(5):1046-51.

[14] Cirrone GAP et al. Hadrontherapy: a Geant4-based tool for proton/ion-therapy studies. Prog Nucl Sci Technol 2011;2:207-12.

[15] http://www.gammex.com/. 